\title{
Análise comparativa da criação dos camarões-rosa Farfantepenaeus brasiliensis e Farfantepenaeus paulensis criados em gaiolas em ambiente estuarino
}

\author{
Comparative analysis of pink shrimp Farfantepenaeus brasiliensis and Farfantepenaeus paulensis \\ reared in estuarine cage culture system
}

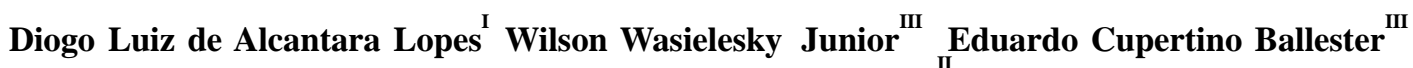 \\ Sílvio Ricardo Maurano Peixoto
}

\section{RESUMO}

A análise da criação de Farfantepenaeus brasiliensis e Farfantepenaeus paulensis em gaiolas, na Lagoa dos Patos, Rio Grande do Sul (RS), foi realizada a partir de juvenis produzidos em cativeiro com peso médio inicial de aproximadamente 1,2g. O experimento foi realizado em seis gaiolas (três gaiolas/ tratamento), com abertura de malha de $5 \mathrm{~mm}$, área de fundo de $4 \mathrm{~m}^{2}$ durante 65 dias. A distribuição dos indivíduos nos tratamentos (F. brasiliensis e F. paulensis) foi aleatória, mantendo-se a densidade de 20 camarões $m^{-2}$ nas unidades experimentais. A cada 15 dias foram realizadas biometrias para ajuste da quantidade de ração fornecida e avaliação do crescimento dos camarões. Ao final do experimento, foi realizada a biometria total dos camarões para avaliação da sobrevivência. Não houve diferença significativa entre a sobrevivência de $\boldsymbol{F}$. brasiliensis $(94,17 \pm 9,04)$ e $\boldsymbol{F}$. paulensis $(98,50 \pm 0,71)$. O peso médio final foi significativamente maior para o F. brasiliensis $(7,98 \pm 0,94 \mathrm{~g})$; porém, não foram observadas diferenças significativas na produção de biomassa $\left(127,81 \pm 17,93\right.$ e 126,65 $\left.\pm 1,74 \mathrm{~g} \mathrm{~m}^{-2}\right)$ e

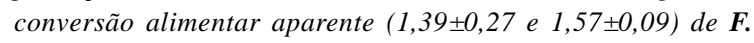
brasiliensis e $\boldsymbol{F}$. paulensis, respectivamente. Os resultados indicam que $\boldsymbol{F}$. brasiliensis apresenta potencial para produção em estruturas alternativas e incentivam que novas pesquisas sejam realizadas para o desenvolvimento de um pacote tecnológico de produção dessas espécies.

Palavras-chave: criação, Farfantepenaeus brasiliensis, Farfantepenaeus paulensis, sistema alternativo, gaiola, crustáceo, decápode, penaeidae.

\begin{abstract}
The cage culture of Farfantepenaeus brasiliensis and Farfantepenaeus paulensis was analyzed in the Patos Lagoon estuary using juveniles produced in captivity. Mean initial weight of the juveniles was approximately $1.2 \mathrm{~g}$. The experiment was conducted in 6 cages (3 per treatment), with mesh size of $5 \mathrm{~mm}$ and bottom area of $4 \mathrm{~m}^{2}$, during 65 days. The individuals were randomly distributed into two treatments ( $\boldsymbol{F}$. brasiliensis e F. paulensis), keeping the stocking density of 20 shrimps $m^{-2}$ in the experimental units (cages). Each 15 days shrimps were weighted to adjust the amount of feed and to evaluate growth. In the end of the experiment, all the shrimp were weighed and counted to determine the survival. Survival did not differ significantly between $\boldsymbol{F}$. brasiliensis $(94.17 \pm 9.04)$ and $\boldsymbol{F}$. paulensis (98.50 \pm 0.71 ). Although the mean final weight was significantly higher for $\mathbf{F}$. brasiliensis $(7.98 \pm 0.94 \mathrm{~g})$, there were no significant differences in terms of total biomass production $\left(127.81 \pm 17.93\right.$ e $\left.126.65 \pm 1.74 \mathrm{~g} \mathrm{~m}^{-2}\right)$ and apparent feed conversion ratio $(1.39 \pm 0.27$ e $1.57 \pm 0.09)$ between $\boldsymbol{F}$. brasiliensis and $\boldsymbol{F}$. paulensis, respectively. The results indicate that $\mathbf{F}$. brasiliensis show potential to be cultured in alternative systems and motivate the development of the technological package for culture of this species in the Patos Lagoon estuary.
\end{abstract}

Key words: Farfantepenaeus brasiliensis, Farfantepenaeus paulensis, alternative system, cages, crustácea, decapoda, penaeidae.

\section{INTRODUÇÃO}

A carcinocultura mundial está baseada principalmente na espécie Litopenaeus vannamei,

\footnotetext{
IDepartamento de Oceanografia, Laboratório de Maricultura, Universidade Federal do Rio Grande (FURG), CP 474, 96201-900, Rio Grande, RS, Brasil. E-mail: diogolalzoo@hotmail.com. Autor para correspondência.

IFURG, Rio Grande, RS, Brasil. Departamento de Pesca e Aqüicultura, Universidade Federal Rural de Pernambuco (UFRPE), Campus Dois Irmãos, Recife, PE, Brasil.

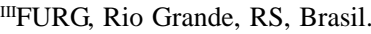


devido a sua rusticidade e à existência de um pacote tecnológico de criação desenvolvido. Essa tendência também ocorre no Brasil, onde essa espécie exótica é amplamente criada em viveiros escavados próximos aos estuários e lagoas costeiras ao longo do litoral. As fazendas de criação de camarões marinhos estão localizadas em áreas adjacentes a estuários, região onde normalmente os Peneídeos realizam grande parte do seu desenvolvimento (fase juvenil). A elevada produtividade natural (primária e secundária presente nesses sistemas) também favorece o crescimento no sistema de criação (PAQUOTTE et al.,1998).

A utilização de viveiros escavados para criação de camarões marinhos dificulta a introdução de novos produtores devido à grande quantidade de capital de investimento, tanto para a aquisição de áreas valorizadas junto ao litoral e construção dos viveiros, como para o manejo durante a produção. Uma das alternativas para integrar a população ribeirinha (pescadores artesanais e pequenos agricultores) na carcinocultura marinha é desenvolver sistemas alternativos de criação que sejam realizados diretamente em ambiente estuarino e que apresentem menor custo, tanto de implantação, como no manejo (WALFORD \& LAM, 1987; WASIELESKY, 2000).

$\mathrm{Na}$ tentativa de desenvolver um pacote tecnológico para a produção de camarão na região estuarina da Lagoa dos Patos, Rio Grande do Sul (RS), Brasil, o Laboratório de Maricultura da Fundação Universidade Federal do Rio Grande (FURG) vem utilizando com sucesso uma espécie nativa da costa brasileira (Farfantepenaeus paulensis) em sistemas alternativos de criação em gaiolas (tanques-rede) e cercados (DOMINGOS, 2000; WASIELESKY et al, (2001, 2003); MEDVEDOVSKY, 2002; VAZ et al., 2004; SILVA, 2003; CAVALCANTI, 2005; KRUMMENAUER et al., 2006). Apesar desses sistemas envolverem menores custos de produção, quando comparados com os sistemas convencionais (WASIELESKY 2000; FINCO et al. (2003), a falta de estudos relacionados à criação de espécies nativas de camarões tem sido um dos gargalos para o desenvolvimento dessa atividade. Segundo MARCHIORI (1996), as principais espécies marinhas de camarões nativos encontrados no Brasil são: L. schimitti, F. paulensis, F. subtilis e $F$. brasiliensis. Os camarões-rosa $\boldsymbol{F}$. brasiliensis e $\boldsymbol{F}$. paulensis se sobrepõem nas suas distribuições, uma vez que a primeira espécie distribui-se desde a Carolina do Norte (EUA) até a costa do RS (D’INCAO, 1999), e a segunda espécie, desde Ilhéus, Bahia, até Buenos Aires, Argentina (D’INCAO, 1991; 1999).

Apesar da carência de estudos relativos à criação em cativeiro, há diversas vantagens em se trabalhar com espécies nativas de camarões do sul do
Brasil, tais como melhor tolerância e crescimento em baixas temperaturas, disponibilidade de reprodutores na região costeira e melhor aceitação no mercado local (SANDIFER et al., 1993). Na busca de outras espécies nativas que possam ser utilizadas na carcinocultura nacional, o presente estudo avaliou o desempenho de $\boldsymbol{F}$. brasiliensis em comparação com $\boldsymbol{F}$. paulensis, em sistemas alternativos de produção, em gaiolas no estuário da Lagoa dos Patos.

\section{MATERIAL E MÉTODOS}

Os juvenis de $\boldsymbol{F}$. brasiliensis e $\boldsymbol{F}$. paulensis foram produzidos na Estação Marinha de Aquacultura da FURG e transportados para a enseada estuarina do Saco do Justino, localizada na Lagoa dos Patos, RS, onde permaneceram em sistema de berçário, em gaiolas até atingir peso médio de 1,15 $\pm 0,34 \mathrm{~g}$ ( $\boldsymbol{F}$. brasiliensis) e 1,23 \pm 0,53g (F. paulensis). Os juvenis foram então transferidos para o sistema de engorda montado nessa mesma localidade, dando início ao experimento (9 de fevereiro de 2006), com duração de 65 dias.

A distribuição dos indivíduos nos dois tratamentos (F. brasiliensis e $\boldsymbol{F}$. paulensis) foi aleatória, mantendo-se a densidade padrão de 20 camarões $\mathrm{m}^{-2}$ nas unidades experimentais (gaiolas). Para cada tratamento, foram utilizadas três gaiolas confeccionadas com malha de poliéster revestida com PVC $\left(\right.$ Sansuy $\left.^{\circledR}\right)$, área de fundo de $4 \mathrm{~m}^{2}$ ( 2 x $\left.2 \mathrm{~m}\right)$ e altura de $2 \mathrm{~m}$, fixadas e suspensas em uma armação de bambus. Nos 30 dias iniciais do experimento, os juvenis foram mantidos em gaiolas com abertura de malha de $1,5 \mathrm{~mm}$, sendo posteriormente transferidos para gaiolas com abertura de malha de $5 \mathrm{~mm}$, onde permaneceram até o final do experimento.

A alimentação foi realizada em alimentadores do tipo bandejas. Para facilitar a alimentação, um cano de PVC de 2,5m foi fixado na bandeja de alimentação e na armação de sustentação das gaiolas, sendo o alimento despejado na bandeja de alimentação através do cano de PVC. A alimentação era fornecida diariamente em uma única refeição ao anoitecer (ração comercial Camaronina ( CR2, 40\% Proteína Bruta).

Aproximadamente a cada 15 dias foram realizadas biometrias parciais (30 camarões por gaiola), para estimar o peso médio dos indivíduos (peso úmido). Nessa oportunidade, a quantidade de ração fornecida foi ajustada de acordo com o peso médio dos indivíduos conforme proposto por WASIELESKY (2000). O registro da temperatura e da salinidade foi realizado semanalmente durante o decorrer do estudo. Ao final do experimento, realizou-se, além da biometria total, a contagem de todos os camarões remanescentes 
nas gaiolas de cada tratamento para avaliação da sobrevivência. Foram analisados o peso final, a taxa de crescimento semanal, a biomassa produzida, sobrevivência e a conversão alimentar aparente de $\boldsymbol{F}$. brasiliensis e $\boldsymbol{F}$. paulensis.

Para o cálculo da taxa de crescimento semanal (TCS), foi utilizada a fórmula:

TCS $=((\mathrm{PF}-\mathrm{PI}) / \mathrm{T}) \times 7$, em que: $\mathrm{PF}=$ peso final, $\mathrm{PI}=$ peso inicial e $\mathrm{T}=$ intervalo de tempo entre biometrias sucessivas (dias).

Na determinação da taxa de sobrevivência final (S), foi utilizada a fórmula:

$\mathrm{S}(\%)=(100 \times \mathrm{NFC}) / \mathrm{NIC}$, em que: $\mathrm{NFC}=$ número final de camarões, NIC = número inicial de camarões.

Para estimar a biomassa total (BTP) de camarão produzido por $\mathrm{m}^{2}$, foi utilizada a fórmula:

BTP = (GP x NTIS) $/$ área de fundo da gaiola em $\mathrm{m}^{2}$, em que: $\mathrm{GP}=$ ganho em peso = (peso final - peso inicial) e NTIS = número total de indivíduos sobreviventes.

A conversão alimentar aparente (CAA) foi calculada por meio da seguinte formula:

$\mathrm{CAA}=\mathrm{QRF} / \mathrm{BTP}$, em que: $\mathrm{QRF}=$ quantidade de ração fornecida e $\mathrm{BTP}=$ biomassa total produzida.
Os valores de peso final, taxa de crescimento semanal, biomassa produzida, sobrevivência e conversão alimentar, nas repetições de cada tratamento, foram primeiramente submetidos à análise de variância (ANOVA), levando em consideração as premissas (normalidade e homogeneidade). Quando não detectadas diferenças significativas $(\mathrm{P}<0,05)$, os valores médios das repetições foram agrupados e submetidos ao teste - $\mathrm{T}$. Valores em percentagem da sobrevivência foram transformados (arco seno da raiz quadrada) para análise estatística, mas estão apresentados na sua forma original.

\section{RESULTADOS}

No decorrer do estudo, as médias ( \pm desvios- padrão) da temperatura e a salinidade da água no estuário da Lagoa dos Patos foram $26^{\circ} \mathrm{C}( \pm 2)$ e 17 $( \pm 2)$, respectivamente. Os valores máximos e mínimos encontrados foram de, respectivamente, 30 e $13^{\circ} \mathrm{C}$ para temperatura e de 22 e 13\% para salinidade. A variação da temperatura e da salinidade estão ilustradas na figura 1. A sobrevivência, biomassa produzida e conversão alimentar aparente não apresentaram diferença estatística significativa $(\mathrm{P}<0,05)$ entre as espécies testadas (Tabela 1). Porém, o peso médio final foi

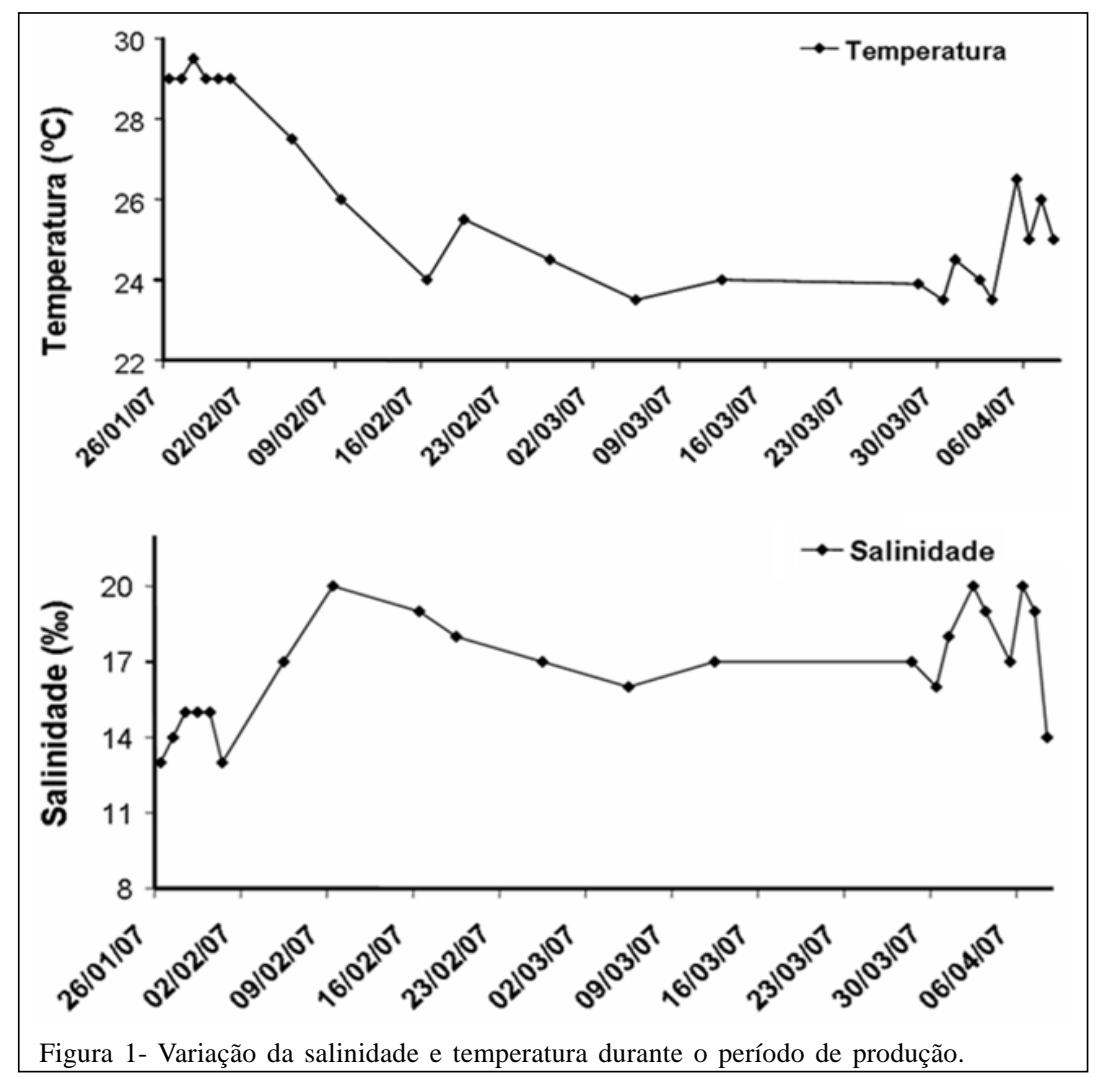

Ciência Rural, v.39, n.5, ago, 2009. 
Tabela 1 - Valores médios ( \pm desvio padrão) de sobrevivência, peso final, biomassa produzida e conversão alimentar aparente (CCA) para Farfantepenaeus brasiliensis e Farfantepenaeus paulensis criados em gaiolas no estuário da Lagoa dos Patos.

\begin{tabular}{lll}
\hline & F. paulensis & F. brasiliensis \\
\hline Sobrevivência (\%) & $98,50 \pm 0,71^{\text {a }}$ & $94,17 \pm 9,04^{\text {a }}$ \\
Peso final (g) & $6,96 \pm 1,12^{\text {a }}$ & $7,98 \pm 0,94^{\mathrm{b}}$ \\
$\begin{array}{l}\text { Biomassa produzida g m } \\
\text { Conversão alimentar }\end{array}$ & $126,65 \pm 1,74^{\text {a }}$ & $127,81 \pm 17,93^{\mathrm{a}}$ \\
$\begin{array}{l}\text { aparente (CAA) } \\
\text { a }\end{array}$ & $1,57 \pm 0,09^{\text {a }}$ & $1,39 \pm 0,27^{\text {a }}$ \\
\hline
\end{tabular}

Letras diferentes na mesma linha indicam que foram verificas diferenças significativas ( $\mathrm{P} \varangle 0,05$ ) entre os tratamentos.

significativamente superior para $\boldsymbol{F}$. brasiliensis (7,95 $\pm 1,01 \mathrm{~g})$, quando comparado com $\boldsymbol{F}$. paulensis (6,96 $\pm 1,12 \mathrm{~g})$, (Tabela 1)

A figura 2 ilustra o peso médio dos camarõesrosa $\boldsymbol{F}$. paulensis e $\boldsymbol{F}$. brasiliensis ao longo do período experimental. Não foi observada diferença significativa $(\mathrm{P}>0,05)$ entre as espécies apenas nos dias 0 e 49; porém, nas demais biometrias (dias 18, 32 e 65), F. brasiliensis apresentou um peso médio significativamente superior à espécie $\boldsymbol{F}$. paulensis $(\mathrm{P}<0,05)$. Os valores da taxa de crescimento semanal no decorrer do estudo não apresentaram diferenças significativas $(\mathrm{P}>0,05)$ entre F. brasiliensis e F. paulensis, (Figura 3).

\section{DISCUSSÃO}

Existe normalmente uma grande variação dos parâmetros físico-químicos da água nos ambientes estuarinos que podem influenciar o crescimento de camarões marinhos. Dentre os parâmetros, a temperatura é um dos que mais afetam o processo de muda e o crescimento dos crustáceos (LOWERY et al., 1988). KUMMENAUER et al. (2006), avaliando a produção de $\boldsymbol{F}$. paulensis no estuário da Lagoa dos Patos durante o período de outono, determinaram que há redução na produção, nos meses mais frios, demonstrando que o período de utilização desse estuário para a produção de camarões deve ser restringido. OLIVERA et al. (1993) afirmam que $\boldsymbol{F}$. paulensis apresenta crescimento mesmo em temperaturas de $15^{\circ} \mathrm{C}$. As temperaturas observadas no presente estudo estão dentro dos limites recomendados por WASIELESKY (2000) e SOARES et al. (2000) para F. paulensis. Estes autores recomendam a faixa entre 16 e $30^{\circ} \mathrm{C}$ para a criação de $\boldsymbol{F}$. paulensis, mas salientam que a temperatura de $27^{\circ} \mathrm{C}$ seria a ideal para o crescimento da espécie. Em virtude de sua distribuição, o camarão-rosa $\boldsymbol{F}$. brasiliensis possivelmente apresente a faixa ótima de temperatura para crescimento com valores superiores ao observado para o F . paulensis; porém, acredita-se que esse parâmetro não tenha influenciado o desenvolvimento da espécie neste estudo. Contudo, são necessários mais estudos para determinar a faixa ideal de temperatura de crescimento de $\boldsymbol{F}$. brasiliensis.

Os valores de salinidade da água no estuário da Lagoa dos Patos, no presente estudo (17 \pm 2$)$, se mantiveram próximos ao limite inferior recomendado por BRITO et al. (2000), para $\boldsymbol{F}$. brasiliensis, e por WASIELESKY (2000), para F. paulensis. VITA et al.,

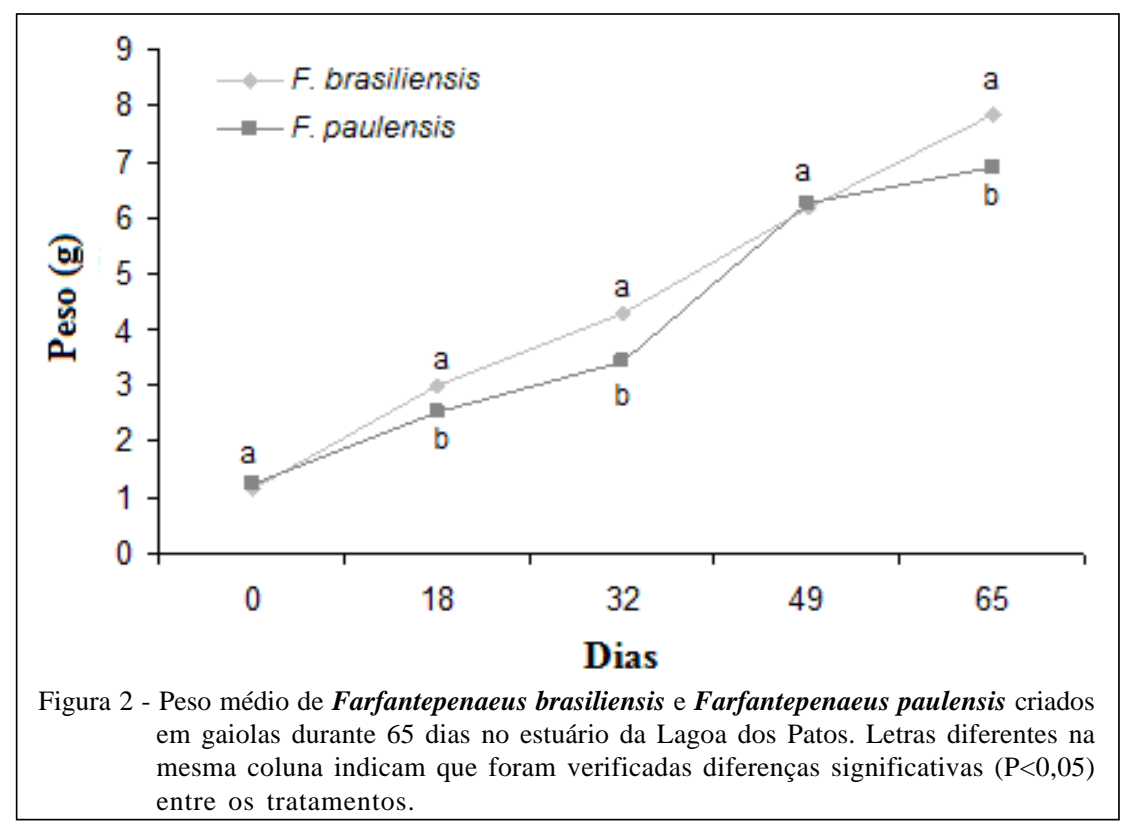

Ciência Rural, v.39, n.5, ago, 2009. 


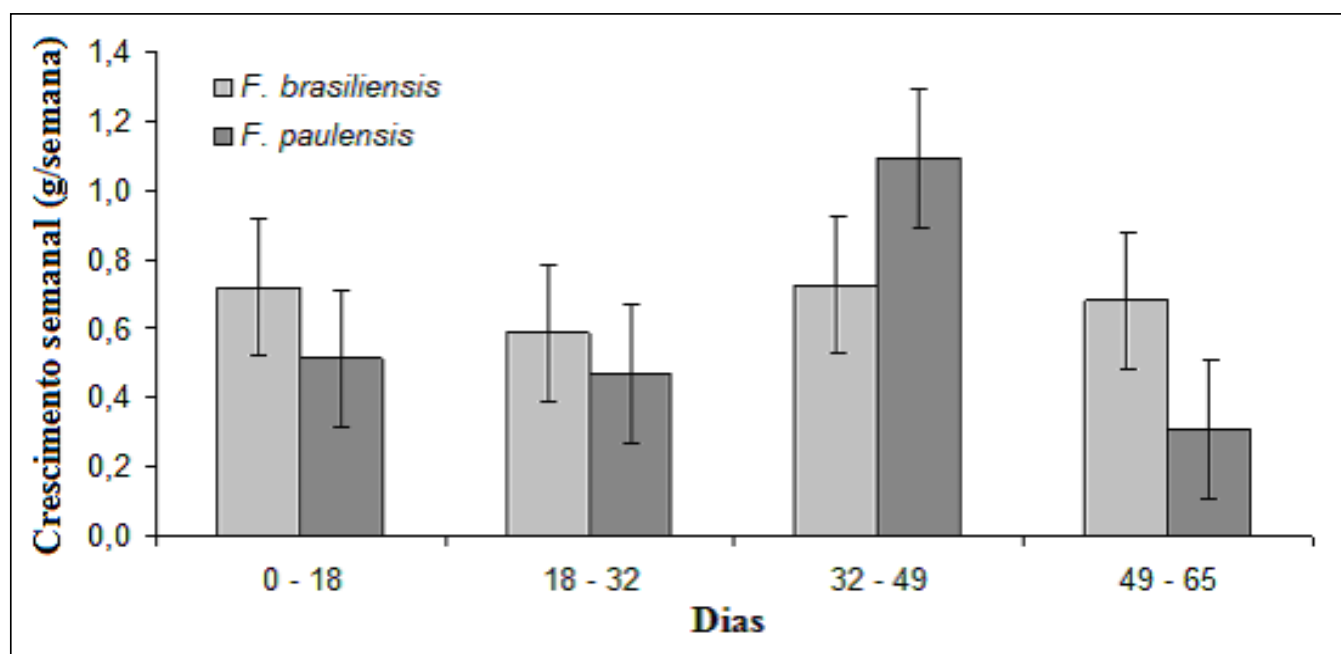

Figura 3 - Taxa de crescimento semanal (g/semana) de Farfantepenaeus brasiliensis e Farfantepenaeus paulensis criados em gaiolas, no estuário da Lagoa dos Patos.

(2006), utilizando irmãos do mesmo lote de $\boldsymbol{F}$. brasiliensis deste estudo, observaram melhor desempenho zootécnico (crescimento e sobrevivência) para $\boldsymbol{F}$. brasiliensis criado em água salobra com salinidade 15. A sobrevivência e o crescimento de $\boldsymbol{F}$. brasiliensis e $\boldsymbol{F}$. paulensis provavelmente não foram afetados pela salinidade no decorrer do estudo.

O peso médio observado durante o estudo, de maneira geral, foi mais elevado para $\boldsymbol{F}$. brasiliensis; porém, esse fator não apresentou reflexo no ganho de peso semanal, na produção de biomassa e na conversão alimentar aparente. MEDVEDOVSKY (2002), criando F. paulensis durante um período de 54 dias em gaiolas, observou que entre as densidades de 15 e 30 camarões $\mathrm{m}^{-2}$ o peso médio final variou de 5,34 a 4,01g e a produção de biomassa de 75,41 a 115,54g m²- Embora os resultados de biomassa tenham sido similares, observamos que o tipo de alimento utilizado neste estudo (ração comercial) foi diferente do utilizado por MEDVEDOVSKY (2002) (rejeito de pesca). As rações comerciais de engorda mais utilizadas no mercado nacional apresentam geralmente um teor de proteína bruta de aproximadamente $35 \%$ e são específicas para L. vannamei. Dessa forma, tem sido recomendada a utilização de rações com teores mais elevados de proteína bruta (>40\%) e/ou complemento alimentar com rejeito de pesca para a criação de espécies de Farfantepenaeus em cativeiro (PEIXOTO et al., 2003).

As taxas de conversão alimentar aparente deste estudo, tanto para $\boldsymbol{F}$. paulensis $(1,81 \pm 0,24)$, quanto para $\boldsymbol{F}$. brasiliensis $(1,75 \pm 0,37)$, foram melhores quando comparadas como os valores encontrados para F. paulensis por SILVA (2003) (2,56 a 3,31) e por
MEDVEDOVSKY (2002) (2,14 a 2,71) utilizando ração comercial e rejeito de pesca. Porém, PRETO (2005), criando $\boldsymbol{F}$. paulensis para isca viva na densidade de 50 camarões $\mathrm{m}^{-2}$ em gaiolas, obteve conversão alimentar de 0,68 , que provavelmente está associada à contribuição do biofilme na alimentação, uma vez que as gaiolas foram colocadas no estuário da Lagoa dos Patos 20 dias antes do povoamento com camarões, e ao ajuste diário da quantidade de ração fornecida.

\section{CONCLUSÃO}

De forma geral, os resultados do presente estudo indicaram que $\boldsymbol{F}$. brasiliensis apresenta potencial para a criação em estruturas alternativas, no estuário da Lagoa dos Patos, nas condições testadas. Embora diversas similaridades tenham sido encontradas, o crescimento semanal e o peso médio observado no decorrer do estudo foram mais homogêneos para o $\boldsymbol{F}$. brasiliensis; porém, não foi observado a diferença significativa na produção de biomassa entre as espécies avaliadas. Os resultados incentivam que, assim como vem ocorrendo com $\boldsymbol{F}$. paulensis, mais estudos precisam ser realizados para fechar o ciclo de vida do F. brasiliensis em cativeiro, determinar as exigências nutricionais, melhorar a conversão alimentar e otimizar o seu desempenho em sistemas alternativos de criação.

\section{REFERÊNCIAS}

BRITO, R. et al. Effect of salinity in survival, growth, and osmotic capacity of early juveniles of Farfantepenaeus brasiliensis (Decapoda: Penaeidae). Jornal of Experimental Marine 
Biology and Ecology, v.244, p. 253-263, 2000. Disponível em: $<\mathrm{ht} \mathrm{t}$ p : / / w w w. s c i e n c e d i r e c t. c o m / science?_ob=ArticleURL\&_udi=B6T8F-3Y9G79T$6 \&$ \&user $=687358 \&$ \&doc $=1 \&$ \&mt $=\&$ \&orig $=$ search $\&$ _sort $=\mathrm{d} \&$ vi e w $=$ c \&_a c ct $=$ C $000037899 \&$ \& version $=1 \&$ _ u r l Versio n $=0 \&$ _ u s e ri d $=687358 \&$ m d 5=a3557bb9fa97f532d09ae7fcbfa28e68>. Doi: 10.1016/S00220981(99)00142-2.

CAVALCANTI, A.M. Efeito da densidade de estocagem sobre o biofilme e o desenvolvimento do camarão-rosa Farfantepenaeus paulensis (Perez-Farfante, 1967) cultivados em cercados na fase de berçário. 2005. 44f. Monografia (graduação no curso de Oceanologia) - Fundação Universidade federal de Rio Grande, Rio Grande, RS.

D’INCAO, F. Pesca e biologia de Penaeus paulensis na Lagoa dos Patos, RS. Atlântica, v.12, p.31-51, 1991.

D'INCAO, F. Subordem DENDROBRANCHIATA (camarões marinhos). In: BUCKUP, L.; BOND-BUCKUP, G. O Crustáceos do Rio Grande do Sul. Porto Alegre, Universidade Federal Rio Grande Sul. 1999. Cap.15, p.275-229.

DOMINGOS, J.A. Cultivo do camarão-rosa Farfantepenaeus paulensis (Decapoda: Penaeidae) em gaiolas utilizando como alimento ração comercial e resíduos de pescado preservados em sal. 2000. 34f. Monografia (graduação do curso de Oceanologia) - Fundação Universidade Federal de Rio Grande, Rio Grande-RS.

FINCO, M.V.A. et al. Viabilidade econômica da criação de camarão em gaiolas e cercados como alternativa de renda à pesca artesanal. Estudos \& Debates, v.10, n.1, p.89-101, 2003.

KRUMMENAUER, D. et al. Viabilidade da criação do camarão rosa Farfantepenaeus paulensis (Crustacea, Decapoda) em gaiolas sob diferentes densidades durante o outono no sul do Brasil. Ciência Rural, v.36, n1, p. 252-257, 2006. Disponível em: $<$ http://www.scielo.br/scielo.php?script=sci_arttext\&pid=S010384782006000100039\&lng=en\&nrm=iso\&tlng=pt $>$. Doi: 10.1590/S0103-84782006000100039.

LOWERY, R.S. Growth, molting and reproduction. In: HOLDICH, D.M.; LOWERY, R.S. (Eds). Freshwater crawfish: biology management and explotation. Portland: Timber, 1988. p.83-113.

MARCHIORI, A.M. Guia ilustrado de maturação e larvicultura do camarão-rosa Penaeus paulensis Perez-Farfante 1967. Rio Grande-RS: Fundação Universidade Federal de Rio Grande, 1996. $79 f$.

MEDVEDOVSKY, K.G. Efeito da densidade de estocagem sobre o crescimento e a sobrevivência do camarão-rosa Farfantepenaeus paulensis (Perez-Farfante, 1967) cultivado em gaiolas no estuário da Lagoa dos Patos, Rio Grande, RS. 2002. 35f. Monografia (graduação no curso de Oceanologia), Fundação Universidade Federal de Rio Grande, Rio Grande, RS.

OLIVERA, A.E. et al. Crescimento do Camarão-rosa Penaeus paulensis no repovoamento da Lagoa de Ibiraquera, Santa Catarina, Brasil. In: SIMPÓSIO BRASILEIRO SOBRE CULTIVO DE CAMARÃO, 1993, João Pessoa. Anais... João Pessoa: MCR Aquacultura, 1993. v.1, p.439-451.

PAQUOTTE, P. et al. Intensive culture of shrimp Penaeus vannamei in floating cages: Zootechnical, Economic and Environmental aspects. Aquaculture, v.164, p. 151-166, 1998. Disponível em: <http://www.sciencedirect.com/ science?_ob=ArticleURL\&_udi=B6T4D-3T 8P28TB\&_user=687358\&_rdoc $=1 \&$ \&fmt $=$ \&_orig $=$ search\&_sort $=$ d\&view $=$ c \&_a c ct $=$ C $000037899 \&$ \& version $=1 \&$ \&_urlVers i o n $=0$ \&_u s e r i d = $687358 \&$ m d $5=$ c 9 246fc2f70bc843aae621bc0d7dcda3>. Doi: 10.1016/S00448486(98)00183-5.

PEIXOTO, S. et al. Comparative analysis of pink shrimp, Farfantepenaeus paulensis, and Pacific white shrimp, Litopenaeus vannamei, culture in extreme southern Brazil. Journal of Applied Aquaculture, v.14, p.101-111, 2003. Disponível em: <http://www.informaworld.com/smpp/ content $\sim \mathrm{db}=$ all ? content $=10.1300 / J 028 v 14 n 01 \_07>$. Doi: 10.1300/J028v14n01_07.

PRETO A.L. Efeito da densidade de estocagem sobre o biofilme e o desempenho do camarão-rosa Farfantepenaeus paulensis cultivado em gaiolas nas fases de berçário e produção de iscas-vivas. 2005. 42f. Dissertação (Mestrado em Aquacultura) - Fundação Universidade Federal de Rio Grande, Rio Grande.

SANDIFER, P. A. et al. Preliminary comparison of the native Penaeus setiferus and the pacific white shrimp Penaeus vannamei for pond culture in South Carolina, USA. Journal at World Aquaculture Society, v.24, p.295-303, 1993. Disponível em: <http://www3.interscience.wiley.com/journal/ 119980316/abstract?CRETRY $=1 \&$ SRETRY $=0>$. Doi: $10.1111 /$ j.1749-7345.1993.tb00161.x.

SILVA, O.A. Cultivo em cercado do camarão-rosa Farfantepenaeus paulensis (Perez-Farfante, 1967) em baixas densidades de estocagem. 2003. 34f. Monografia (graduação no curso de Oceanologia) - Fundação Universidade Federal de Rio Grande, Rio Grande.

SOARES, R.B. et al. Growth and food consuption of pink shrimp Farfantepenaeus paulensis postlarvae under different temperatures. Aquaculture America Book of Abstracts. v.1, p.307, 2000.

VAZ, J.L. et al. Crescimento e sobrevivência de pós-larvas de camarão-rosa (Farfantepenaeus paulensis) em gaiolas e cercados. Scientia Agrícola, v.61, n.3, p.332-335, 2004.

VITA, G. et al. Crescimento, consumo alimentar e sobrevivência de juvenis do camarão-rosa Farfantepenaeus brasiliensis em diferentes salinidades. In: CONGRESSO AQUACIÊNCIAS 2006, Bento Gonçalves, RS, Brasil. Anais... Bento Gonçalves, FURG. 2006.

WALFORD, J; LAM, T.J. Floating hatchery and net cage culture of Penaeus indicus in the Straits of Johore, Singapore. Aquaculture, v.62, p.11-32, 1987. Disponível 
e m : < h t t p :// w w w. sciencedirect.co m / science?_ob=ArticleURL\&_udi=B6T4D-49NPMCP $19 F \&$ \&user $=687358 \& \_r d o c=18 \_\mathrm{fmt}=$ \&_orig $=$ search\&_sort $=\mathrm{d} \& \mathrm{view}=$ c \&_a c ct $=$ C $000037899 \&$ \&_version $=1 \&$ \&_urlVer

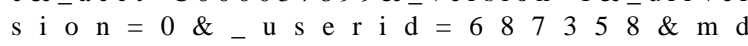
5=7074c1ce6d9719a9eedd6402598b8b0f $>$. Doi: 10.1016/ 0044-8486(87)90181-5.

WASIELESKY, W.J. Cultivo de juvenis do camarão-rosa Farfantepenaeus paulensis (Decapoda, Penaeidae) no estuário da Lagoa dos Patos: efeitos dos parâmetros ambientais. 2000. 194f. Tese (Doutorado em Oceanografia Biológica) - Fundação Universidade Federal de Rio Grande, Rio Grande. 2000.

WASIELESKY W.J. et al. Effect of stocking density on pen reared pink shrimp Farfantepenaeus paulensis (Perez-Farfante, 1967) (Decapoda: Penaeidae). Nauplius, v.9, p.163-167, 2001 